\title{
РАЗРАБОТКА АВТОМАТИЗИРОВАННОЙ СИСТЕМЫ МОНИТОРИНГА ЗДОРОВЬЯ И ОПОВЕЩЕНИЯ ДЛЯ НЕОНАТАЛЬНОЙ ИНТЕНСИВНОЙ ТЕРАПИИ С ИСПОЛЬЗОВАНИЕМ СЕНСОРНЫХ МОБИЛЬНЫХ ОБЛАЧНЫХ ВЫЧИСЛЕНИЙ (СМОВ)
}

\section{DEVELOPMENT OF AN AUTOMATED HEALTH MONITORING SYSTEM AND ALERTS FOR NEONATAL INTENSIVE THERAPY USING SENSORED MOBILE CLOUD COMPUTING (CMOS)}

\section{Hassanin Hatem Mohamed Abdel Maksoud}

Summary. Currently, there is an increase in information and telecommunication technologies in many spheres of life. One of the promising systems that are being created and improved are information automated health monitoring systems.

Most of the currently available medical monitoring and control devices are wired, which limits the freedom of the work environment. Touchscreen mobile cloud computing is the best alternative in such an environment [1].

The neonatal intensive care unit is used to care for sick and premature infants. Hypothermia is an independent risk factor for neonatal mortality and morbidity. To prevent this, an automated monitoring system is needed.

With the introduction of automated health monitoring and alert systems for neonatal intensive care using touch-sensitive mobile cloud computing, a healthcare professional can continuously monitor and access this data via a mobile device using a newborn monitoring app.

When an abnormal situation occurs, a warning is generated on the mobile device of a healthy person. By alerting the healthcare provider with this automated system, affected babies are treated early and the likelihood of recovery is increased.

Thus, neonatal monitoring refers to the monitoring of vital physiological parameters of premature and term infants in critical condition [2].

Keywords: touchscreen mobile cloud computing, neonatal monitoring, physiological parameters of premature, mobile cloud computing, SMOV, WISE systems, WSN.
Хассанин Хатем Мохамед Абдель Максуд Начиональный исследовательский Томский политехнический университет; Хунаньский университет искусств и наук, Провинция Хунань,

Kumaŭ

Blombygg1@gmail.com

Аннотация. В настоящее время наблюдается рост информационных и телекоммуникационных технологий во многих сферах жизнедеятельности. Одной из перспективных систем, которые создаются и совершенствуются, являются информационные автоматизированные системы мониторинга здоровья.

Большинство доступных в настоящее время медицинских устройств для мониторинга и контроля являются проводными, что ограничивает свободу рабочей среды. Сенсорные мобильные облачные вычисления — лучшая альтернатива в такой среде [1].

Отделение интенсивной терапии новорожденных используется для ухода за больными и недоношенными новорожденными. Гипотермия - независимый фактор риска неонатальной смертности и заболеваемости. Для предотвращения этого необходима автоматизированная система мониторинга. При внедрении автоматизированных систем мониторинга здоровья и оповещения для неонатальной интенсивной терапии с использованием сенсорных мобильных облачных вычислений медицинский работник может постоянно контролировать и получать доступ к этим данным через мобильное устройство с помощью приложения для наблюдения за новорожденными.

Возникновении нештатной ситуации на мобильном устройстве здорового человека генерируется предупреждение. Предупреждая медицинского работника с помощью такой автоматизированной системы, пострадавшим младенцам оказывается помощь на раннем этапе и повышается вероятность выздоровления.

Таким образом, неонатальный мониторинг относится к мониторингу жизненно важных физиологических параметров недоношенных и доношенных детей, находящихся в критическом состоянии [2].

Ключевые слова: сенсорные мобильные облачные вычисления, неонатальный мониторинг, физиологических параметров недоношенных, мобильные облачные вычисления, CMOB, WISE системы, WSN. 
B рамках выполнения работы предлагается использование сенсорных мобильных облачных вычислений. Мобильные облачные вычисления (МОВ) предоставляют богатые вычислительные ресурсы мобильным пользователям, операторам сетей и поставщикам облачных вычислений.

СМОВ - это сочетание облачных вычислений, мобильных вычислений и беспроводных сетей. Преимущества СМОВ перед облачными вычислениями:

1. Гибкость: благодаря гибкости пользователи могут получать доступ к данным с помощью своих устройств из любой точки мира.

2. Доступность данных: доступность данных позволяет пользователю получить доступ к своим данным в любое время.

3. Несколько платформ: МОВ также обеспечивает поддержку нескольких платформ.

Текущая архитектура мобильных облачных вычислений включает следующие компоненты:

1. Региональный центр обработки данных (RDC);

2. Ядро беспроводной связи;

3. Базовые станции.

Текущая архитектура представлена на рисунке 1. Архитектура МОВ позволяет пользователям переносить свои операции в облако[3].

RDC состоит из различных устройств безопасности, источников питания, средств управления средой и т.д. Облачные центры обработки данных расположены в разных местах по всему миру[4].

Удаленный мониторинг здоровья был предложен и исследовался в течение нескольких десятилетий[5]. Датчики и сенсорные сети - это фундаментальные технологии, которые используются для сбора ряда сигналов, связанных со здоровьем. Типичным решением для удаленного мониторинга состояния здоровья является использование смартфона в качестве сетевого шлюза, который собирает сигналы датчиков с дальнейшей обработкой или без нее, а затем передает их в соответствующий сервер обработки данных.

Другой ключевой концепцией является сеть Body Area Network (BAN), которая состоит из ряда взаимосвязанных носимых сенсорных устройств для сбора ряда биомедицинских параметров, таких как ЭКГ, ЭЭГ, артериальное давление и температура тела. Такие узлы датчиков связаны между собой с помощью различных протоколов связи, таких как ZigBee, Bluetooth и WiFi.

Из-за ограниченных вычислительных возможностей сенсорных устройств, которые не могут манипулиро- вать всеми считываемыми данными, типичным решением является использование смартфона или планшета в качестве компонента обработки данных. С помощью смартфона или планшета можно выполнить ряд задач, например, обработку необработанных данных, визуализацию данных для пользователей и передачу данных в другие центры обработки данных. Пациенты, врачи и члены семьи могут получить доступ к данным через Интернет или мобильное приложение. В качестве примера системы мониторинга рассмотрим структуру носимой ІоТ-облачной системы мониторинга здоровья (WISE), в которой используется ряд взаимосвязанных носимых датчиков для наблюдения за состоянием здоровья объекта[6].

Можно получить набор биомедицинских сигналов, включая артериальное давление, сердцебиение и температуру тела. Из-за ограниченной памяти и вычислительной мощности узлов датчиков, а также во избежание принятия смартфона в качестве блока обработки данные датчиков, собранные с этих носимых датчиков, будут передаваться непосредственно на облачный сервер.

Общая архитектура WISE системы показана на рисунке 2. Система WISE содержит три основных компонента: сеть WISE body area network (W-BAN), облако WISE (W-Cloud) и пользователя WISE.

W-BAN состоит из трех категорий считывающих устройств, которыми являются датчики пульса, датчики температуры тела, и датчик артериального давления.

Данные датчика сердцебиения указывают на регулярность сердцебиения, которая также может отражать активность миокарда. Датчик сердцебиения обеспечивает простой способ изучения функции сердца, он отслеживает поток крови через мочку уха.

Поскольку сердце проталкивает кровь по кровеносным сосудам в мочке уха, количество крови в ухе меняется со временем. Датчик пропускает световой поток (небольшую лампу накаливания) через ухо и измеряет передаваемый свет. Датчик также можно использовать на кончике пальца или на перепонке кожи между большим и указательным пальцами.

Датчик температуры считывает температуру и показывает температуру в градусах Цельсия. LM35 - это низковольтная ИС, которая потребляет около +5 В постоянного тока. Датчик артериального давления - это неинвазивный датчик, предназначенный для измерения артериального давления человека. Он измеряет систолическое, диастолическое и среднее артериальное давление с помощью осциллометрического метода[7]. 


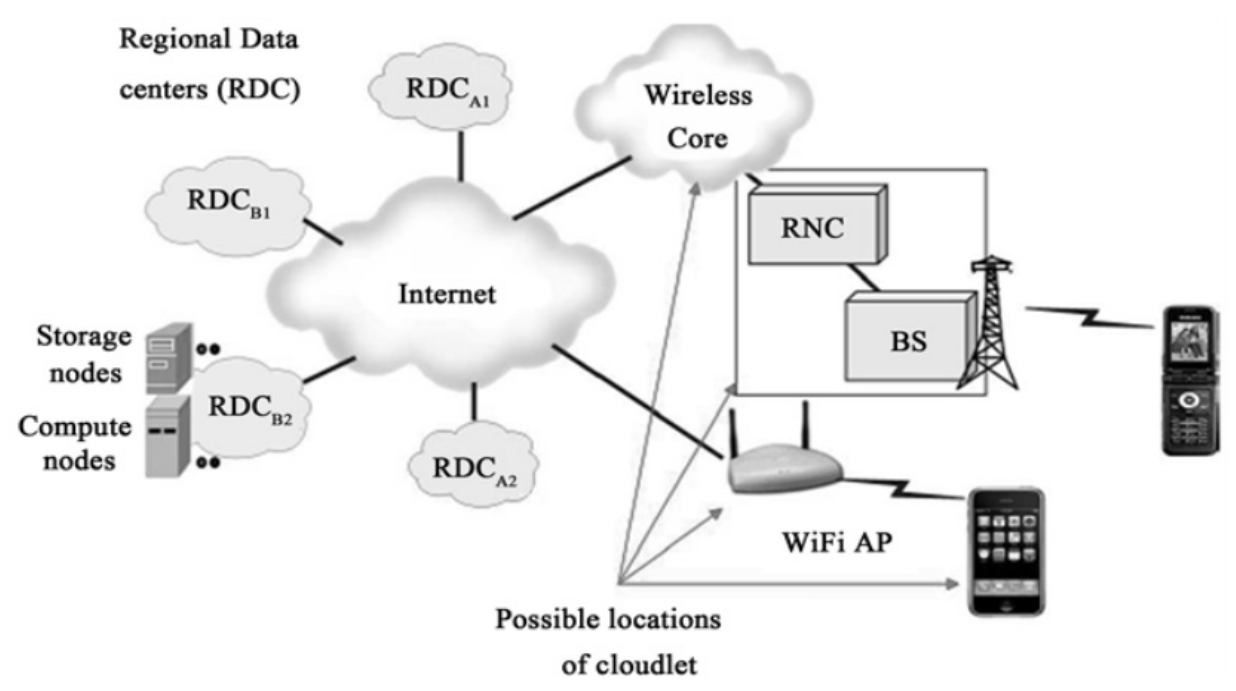

Рис. 1. Текущая архитектура мобильных облачных вычислений

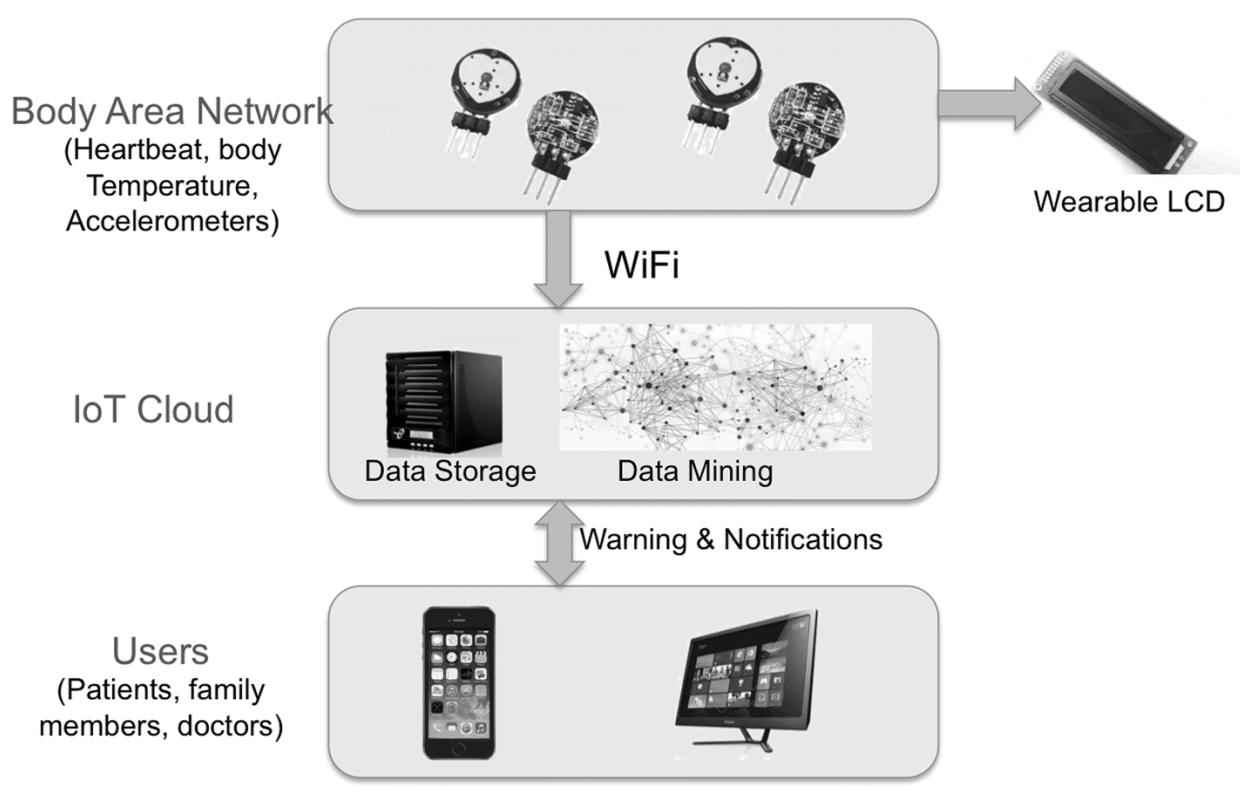

Рис. 2. Общая архитектура WISE системы

Артериальное давление не всегда остается неизменным. Оно меняется в соответствии с потребностями тела. На него влияют различные факторы, включая положение тела, дыхание или эмоциональное состояние, упражнения и сон[8]. Однако с WISE данные из W-BAN можно передавать в облако через Wi-Fi напрямую, без использования смартфона[9]. Представленная в качества примера система WISE разработана на основе сенсорной платформы Arduino, интегрированной с вышеупомянутыми сенсорными узлами. Архитектура реализация WISE системы представлена на рисунке 3.
В дополнение к датчикам, встроенным в WISE, также используются несколько компонентов. Во-первых, портативный считыватель RFID подключается к платформе Arduino, что облегчает идентификацию различных пользователей, поэтому метка RFID должна составляться для каждого отдельного пользователя.

Легкий ЖК-дисплей включен в качестве альтернативной опции для доступа пользователя к данным, как показано на рисунке 4. Кроме того, WISE также оснащен модулем WiFi, который позволяет передавать данные в облако, а затем позволяет авторизованным пользова- 


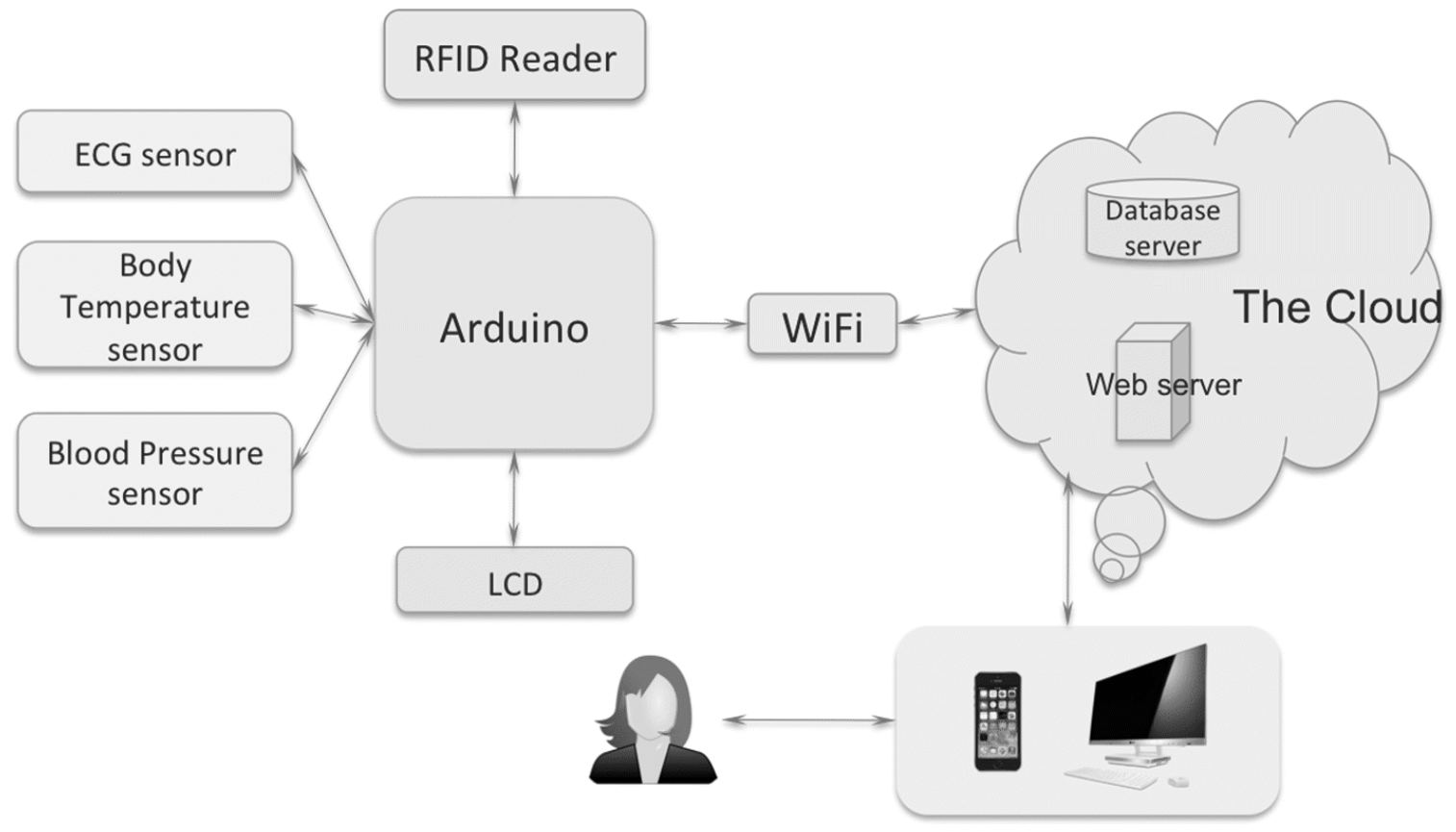

Рис. 3. Архитектура системы WISE

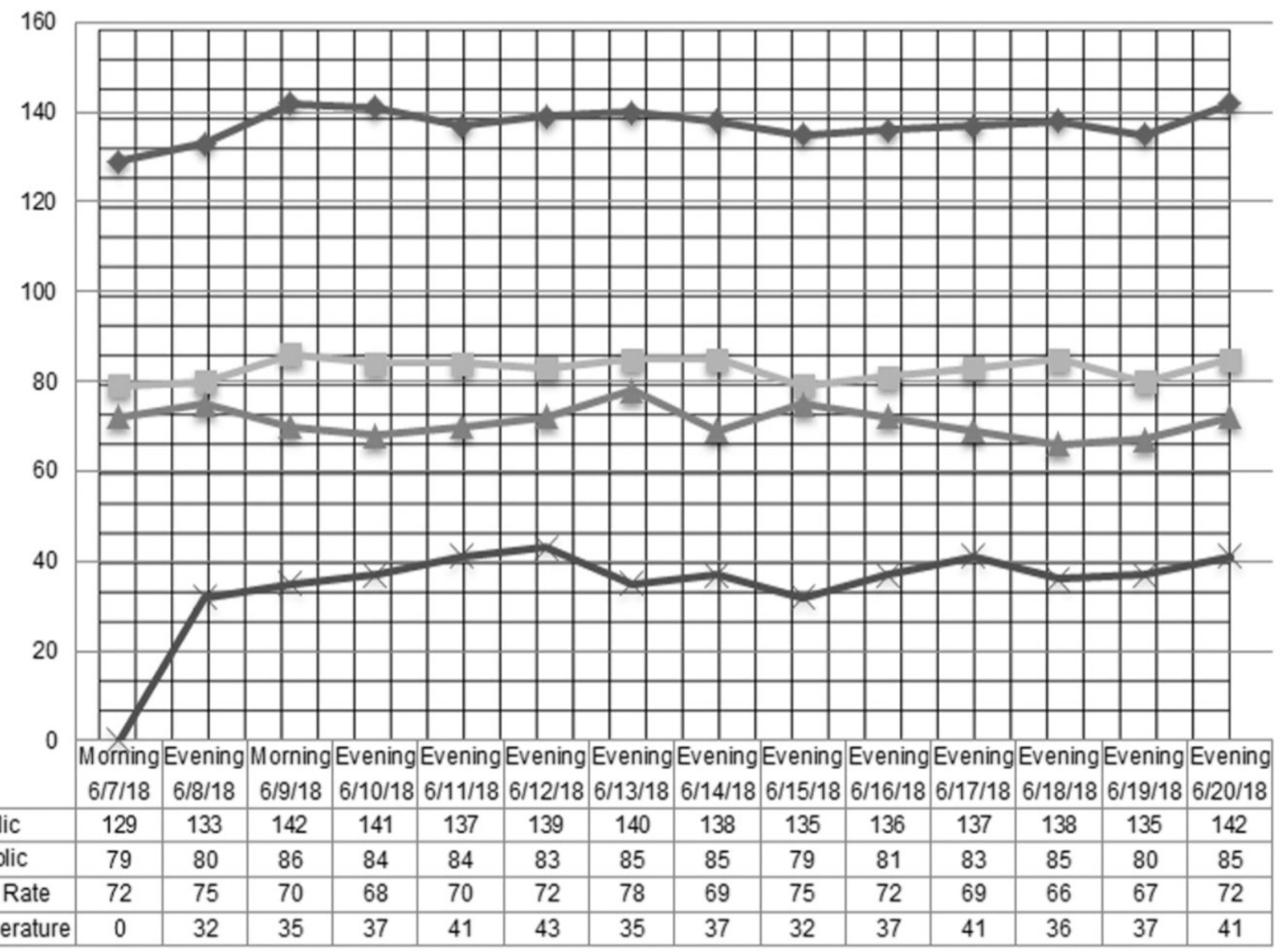

Рис. 4. Исторические данные состояния здоровья пациента 
телям получать доступ к данным в реальном времени из любого места в любое время.

С развитием технологий облачных вычислений данные WISE BAN могут храниться и эффективно обрабатываться в облаке. Поэтому применяется механизм фильтрации данных, чтобы избежать недействительных данных. Более того, для выявления и точного диагноза потенциального заболевания часто требуется определенный объем исторических данных; поэтому в WISE-Cloud создается облачная база данных для хранения данных датчиков из WISE-BAN для каждого отдельного пользователя;

- Визуализация данных. Реализована веб-схема визуализации данных для авторизованных пользователей для доступа к данным;

- Выявление и уведомление о заболевании. Внезапный сердечный приступ часто серьезно угрожает жизни человека, страдающего сердечными заболеваниями. WISE стремится защитить пациентов от таких травм, и важно, чтобы состояние здоровья пациентов можно было контролировать и понимать. Более того, любое подозрительное или ненормальное показание датчика может быть идентифицировано, и уведомления могут быть отправлены идентифицированным пользователям, таким как члены семьи и врачи.

После подачи питания определяется температура термистора и частота пульса на датчике счетчика импульсов. Та же информация загружается на сервер базы данных WISE-Cloud, а затем отображается на веб-странице в режиме реального времени.

Аналогичный механизм также применяется к датчику артериального давления и данных. Такая информация имеет основополагающее значение для пользователей, позволяющих самостоятельно контролировать состояние своего здоровья, а для врачей - для диагностики потенциальных заболеваний.

Если обнаружено какое-либо ненормальное состояние, для определенной заинтересованной стороны будет сгенерировано предупреждение, которое включает текстовое сообщение для врачей или членов семьи, а предупреждение отображается на ЖК-дисплее для самих пользователей. Кроме того, долгосрочные исторические данные также могут быть визуализированы в облаке, как показано на рисунке 4, на котором показаны данные о сердцебиении и температуре тела за период в несколько недель для конкретного пользователя. Кроме того, аналогичные подходы применяются и для данных датчика артериального давления.

Модуль интеллектуального анализа данных - ключевой компонент для диагностики сердечных заболева- ний. После извлечения ценных характеристик из данных сердцебиения и температуры тела применяются подходы на основе машинного обучения, такие как SVM и нейронная сеть, для создания моделей принятия решений[10].

Важно отметить, что в литературе освящено несколько примеров создания подобных автоматизированных систем мониторинга здоровья пациентов, однако для пациентов неонатальной интенсивной терапии подобные системы мониторинга практически отсутствуют. Это подтверждает актуальность настоящего исследования.

На основании проведенного обзора литературы по теме исследования представим разработку системы мониторинга здоровья и оповещения для неонатальной интенсивной терапии с использованием сенсорных мобильных облачных вычислений.

Особенностью создания СМОВ является то, что в СМОВ обработка данных датчиков выполняется внутри облака. Поскольку мы знаем, что профилактика лучше лечения, ранняя диагностика заболеваний всегда обеспечивает лучшее качество жизни. В большинстве медицинских учреждений по-прежнему используется проводная среда.

Отделение неонатальной помощи, как сказано в [11] и [12] является такой средой, где вместо проводных соединений можно использовать беспроводные датчики.

Текущая система диагностики получает доступ к жизненно важным параметрам через большое количество проводов. Врач или медсестра контролируют эти параметры вручную и предпринимают необходимые действия.

Следовательно, требуется альтернатива для мониторинга критических параметров. Оборудование в отделениях неонатальной интенсивной терапии в основном используется для контроля температуры, особенно для предотвращения переохлаждения новорожденного. Потеря тепла у новорожденных, особенно недоношенных, происходит быстро.

Оборудование интенсивной терапии обеспечивает среду, позволяющую контролировать температуру тела новорожденного.

В странах с умеренным климатом или в зимний сезон младенцев помещают под тепло на некоторое время после рождения, чтобы обеспечить термонейтральную среду (TNE).

Обогреватель определяет температуру тела через проводной датчик, прикрепленный к коже, и отобража- 
Таблица 1. Диапазон температуры тела новорожденных по данным Всемирной организации

здравоохранения

\begin{tabular}{|l|l|}
\hline Тип температуры & Температурный диапазон, ${ }^{\circ} \mathbf{C}$ \\
\hline норма & $36,5-37,5$ \\
\hline легкое переохлаждение & $36-36,4$ \\
\hline умеренное переохлаждение & $32-35,9$ \\
\hline сильное переохлаждение & $<32$ \\
\hline гипертермия & $>37,5$ \\
\hline
\end{tabular}

Таблица 2. Рекомендуемая температура окружающей среды

\begin{tabular}{|c|c|c|c|c|}
\hline \multirow{2}{*}{ Масса новорожденного, г } & \multicolumn{4}{|c|}{ Рекомендуемая температура окружающей среды } \\
\hline & $35^{\circ} \mathrm{C}$ & $34^{\circ} \mathrm{C}$ & $33^{\circ} \mathrm{C}$ & $32^{\circ} \mathrm{C}$ \\
\hline$<1500$ & $1-10$ дней & 11 дней - 3 недели & 3-5 недель & $>5$ недель \\
\hline 1500-1999 & NA & 1-10 дней & 11 дней - 4 недели & $>4$ недель \\
\hline $2000-2499$ & NA & $1-2$ дня & 3 дня - 3 недели & $>3$ недель \\
\hline$>2500$ & NA & NA & $1-2$ дня & $\geq 3$ дней \\
\hline
\end{tabular}

ет измеренную температуру на панели дисплея. Температуру можно контролировать, увеличивая или уменьшая интенсивность источника тепла.

Одновременно меняется и температура окружающей среды. Для предотвращения перегрева необходим постоянный контроль.

Диапазон температуры тела новорожденных по данным Всемирной организации здравоохранения представлен в виде таблицы 1.

TNE - это диапазон внешних температур, при котором потребление кислорода, основная скорость метаболизма, будет наименьшей в зависимости от веса ребенка[13].

Таблица 2. показывает рекомендуемую температуру окружающей среды, которую необходимо поддерживать для TNE.

По степени тяжести переохлажденный ребенок в основном поступает к неонатологу в соответствии с четырьмя состояниями:

- Состояние 1: Первоначально, когда ребенок находится в легкой гипотермии, то есть в переохлаждении, могут возникнуть следующие признаки, такие как синюшность пальцев рук и ног, холодные руки и ноги и т.д. Все эти симптомы в основном появляются из-за сужения периферических сосудов. Иногда ребенок может непрерывно плакать или чувствовать раздражительность, что может быть ранним проявлением поражения нервной системы из-за переохлаждения.
- Состояние 2: если переохлаждение не проходит, это может вызвать угнетение нервной системы. Это проявляется в уменьшении подвижности тела, снижении частоты пульса, гипотонии, плохом сосании груди, непреодолимом крике и рвоте. Учащение дыхания и беспокойство проявляются при повышении давления в легочной артерии.

- Состояние 3: если гипотермия продолжается еще несколько периодов, это может привести к серьезным последствиям, которые характеризуются снижением уровня сахара и насыщения крови кислородом, повышенным потенциальным водородом (РН) крови и т.д. Как правило, коагулопатия может привести к стойкой легочной болезни и гипертония новорожденного. В большинстве тяжелых случаев острая почечная недостаточность увеличивает неонатальную смертность.

- Состояние 4. В некоторых случаях стойкий хронический холодовой стресс приводит к задержке роста из-за снижения анаболизма.

Судороги характеризуются быстрым и многократным сокращением и расслаблением мышц тела. Это проявляется неконтролируемым движением всего тела или каких-либо частей тела. Судороги - важнейший симптом судорожного расстройства. Следовательно, термин судорога иногда используется как синоним припадка. Однако все судорожные расстройства могут не приводить к судорогам, таким как легкие судороги. В этом случае увеличение частоты сердечных сокращений является одним из наиболее важных ключей к обнаружению судорог. Все судороги, такие как симптом, т.е. нервозность, могут не возникать из-за судорожного расстройства. 
Рассмотрим предлагаемую систему мониторинга здоровья и оповещения для неонатальной интенсивной терапии с использованием сенсорных мобильных облачных вычислений.

Принцип работы системы на основе описывается следующими основными положениями:

1. Датчик температуры $\left(S_{1}\right)$, датчик ускорения $\left(S_{2}\right)$ и датчик измерения частоты сердечных сокращений $\left(\mathrm{S}_{3}\right)$ прикреплены к поверхности кожи новорожденного с помощью силиконового неаллергенного ремня. Эти датчики измеряют температуру тела, ускорение движения тела и частоту сердечных сокращений новорожденного.

Второй датчик температуры $\left(\mathrm{S}_{4}\right)$ устанавливается на подставке между детской кроваткой и источником тепла лучистого обогревателя. Этот датчик используется для измерения температуры окружающей среды [14].

2. Эти датчики после измерения соответствующего статуса объекта отправляют значение на базовую станцию датчика, действующую как узел приемника. Узел-получатель отправляет эти значения на компьютер, работающий как блок мониторинга данных, через кабель для передачи данных.

3. Компьютер принимает вес и возраст ребенка в качестве входных данных через программное обеспечение. С компьютера все эти данные, включая состояние работоспособности, отправляются в облако на стороне сервера через точку беспроводного доступа. Внутри облака данные хранятся и обрабатываются.

4. Медицинский работник получает доступ к этим данным внутри облака с помощью приложения для неонатального мониторинга, установленного в его или ее мобильном телефоне. Поскольку данные датчиков, собранные с помощью сенсорных сетей, хранятся и обрабатываются в облаке, а специалисты здравоохранения получают доступ к этим данным с помощью своих мобильных устройств, предлагаемая система называется CMOB, то есть системой на основе СМОВ.

5. Компьютер, работающий в качестве блока мониторинга данных, устанавливает желаемую температуру окружающей среды в соответствии с весом и возрастом ребенка, как показано в таблице 2.

Затем проверяется, находится ли температура тела от 36,5 до $37,5^{\circ} \mathrm{C}$. Также проверяется, не вышла ли температура окружающей среды за пределы, указанные в таблице 2.

6. В обоих случаях, если возникнет какая-либо неблагоприятная ситуация, программное обеспечение уведомит об этом. В то же время, обнаружи- вая отклонения от нормы в данных, хранящихся в облаке, на мобильный телефон медицинского работника генерируется предупреждение, чтобы он или она могли принять надлежащие меры.

Следовательно, не только блок мониторинга данных, но и мобильное устройство используется для информирования медицинского работника о необходимости предпринять какие-либо действия при возникновении каких-либо трудностей. Может случиться так, что перед блоком мониторинга данных никого нет. В таком случае оповещение генерируется на мобильном телефоне здорового человека; в случае возникновения неблагоприятной ситуации медицинский работник автоматически извещается.

7. Информировав медицинского работника, можно устранить любую механическую неисправность, например, если произойдет внезапное повышение уровня излучаемого тепла, которое будет мгновенно обнаружено датчиком и уведомлено в системе, чтобы принять незамедлительные меры для предотвращения перегрева.

8. Может возникнуть ситуация, когда медицинский работник не может прибыть вовремя, и температура должна измениться. В таких случаях лучистый обогреватель работает в режиме сервоуправления для изменения температуры.

В этом случае после генерации предупреждения таймер устанавливается на 60 с. Если таймер истекает, но медицинский работник не приезжаeт, W-RW изменяет температуру, работая в режиме сервоуправления.

9. Движение тела ребенка определяется датчиком ускорения. Если произойдет резкое повышение значения параметра ускорения, то это будет автоматически уведомлено в системе. Затем медицинский работник примет необходимые меры.

10. Частота сердечных сокращений ребенка измеряется датчиком измерения частоты сердечных сокращений. Если обнаружена какая-либо проблема с сердцем, она будет автоматически уведомлена в системе. Соответственно, медицинский работник примет необходимые меры. Следовательно, в системе постоянно отслеживаются температура, ускорение и частота сердечных сокращений. Если температура, ускорение или частота сердечных сокращений кажутся ненормальными, система автоматически уведомляет медицинского работника через его или ее мобильный телефон в любом месте в любое время с помощью приложения. Затем медицинский работник предпринимает необходимые действия.

11. Предлагаемая система основана на WSN, мобильной сети и облаке. Транспортный протокол 


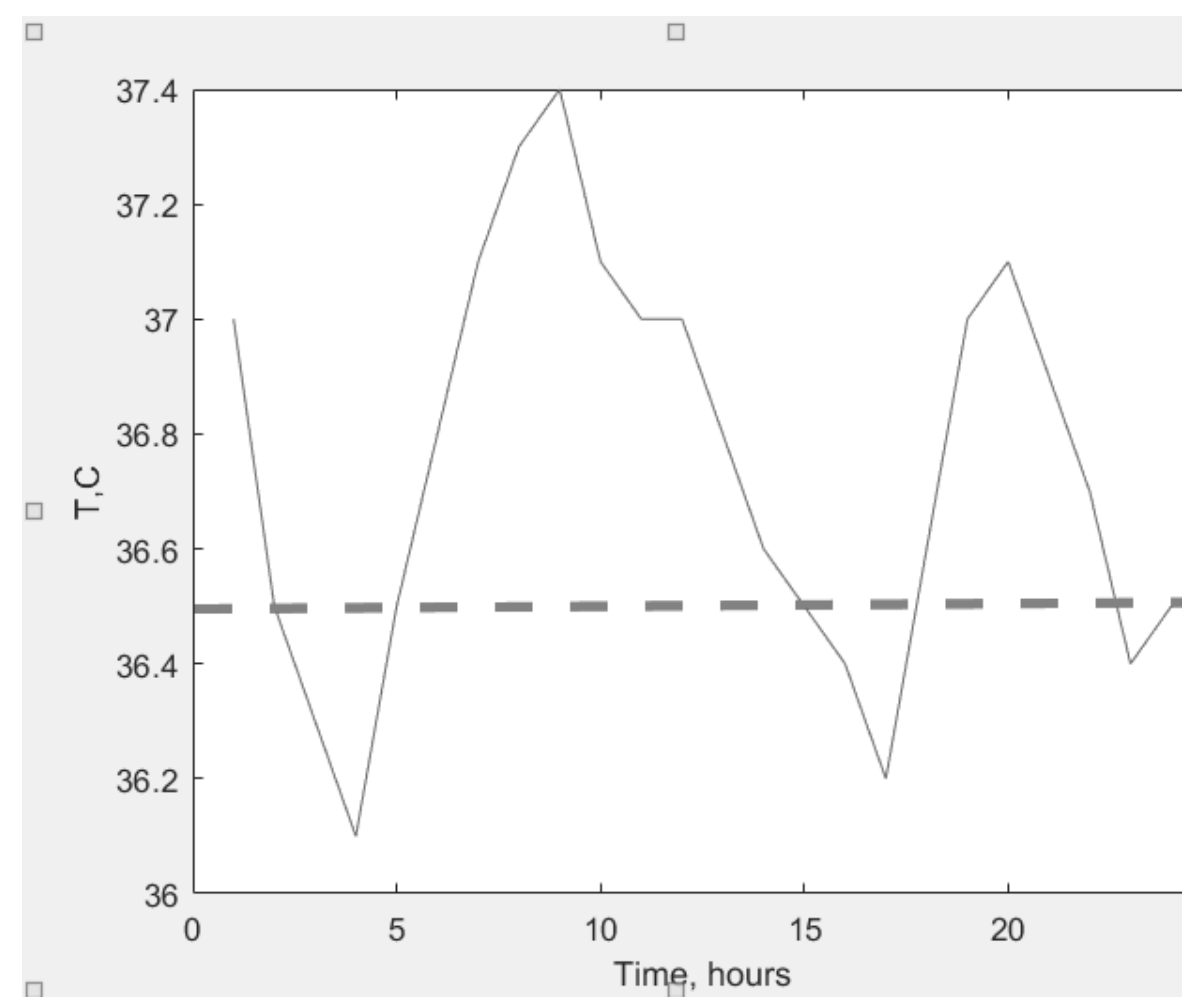

Рис. 5(а). Результаты мониторинга температуры тела ребенка для случая 1. Ниже красной отметки посылается сигнал медицинскому работнику

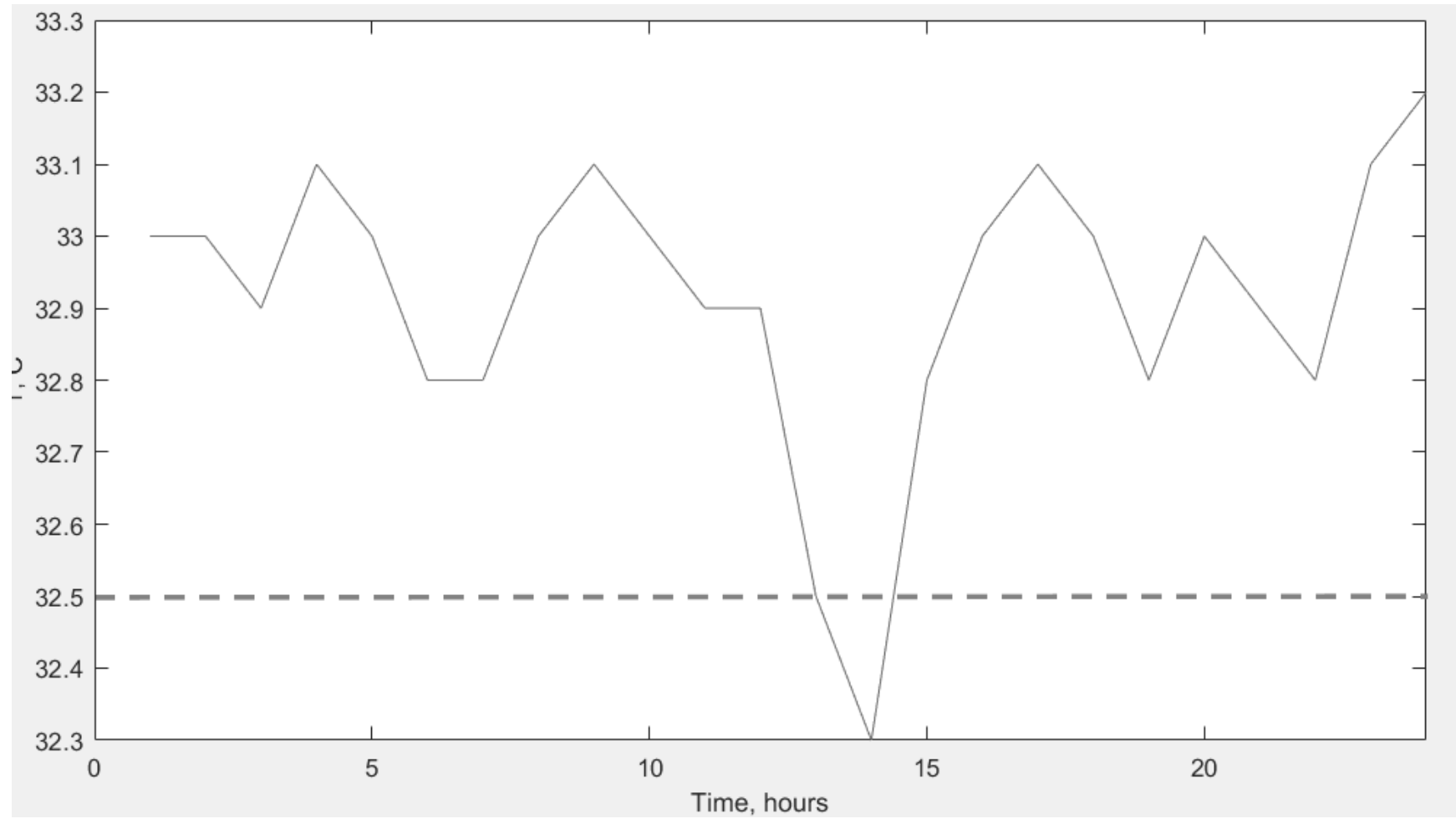

Рис. 5(б). Результаты мониторинга температуры окружающей среды для случая 1. Ниже красной отметки посылается сигнал медицинскому работнику 


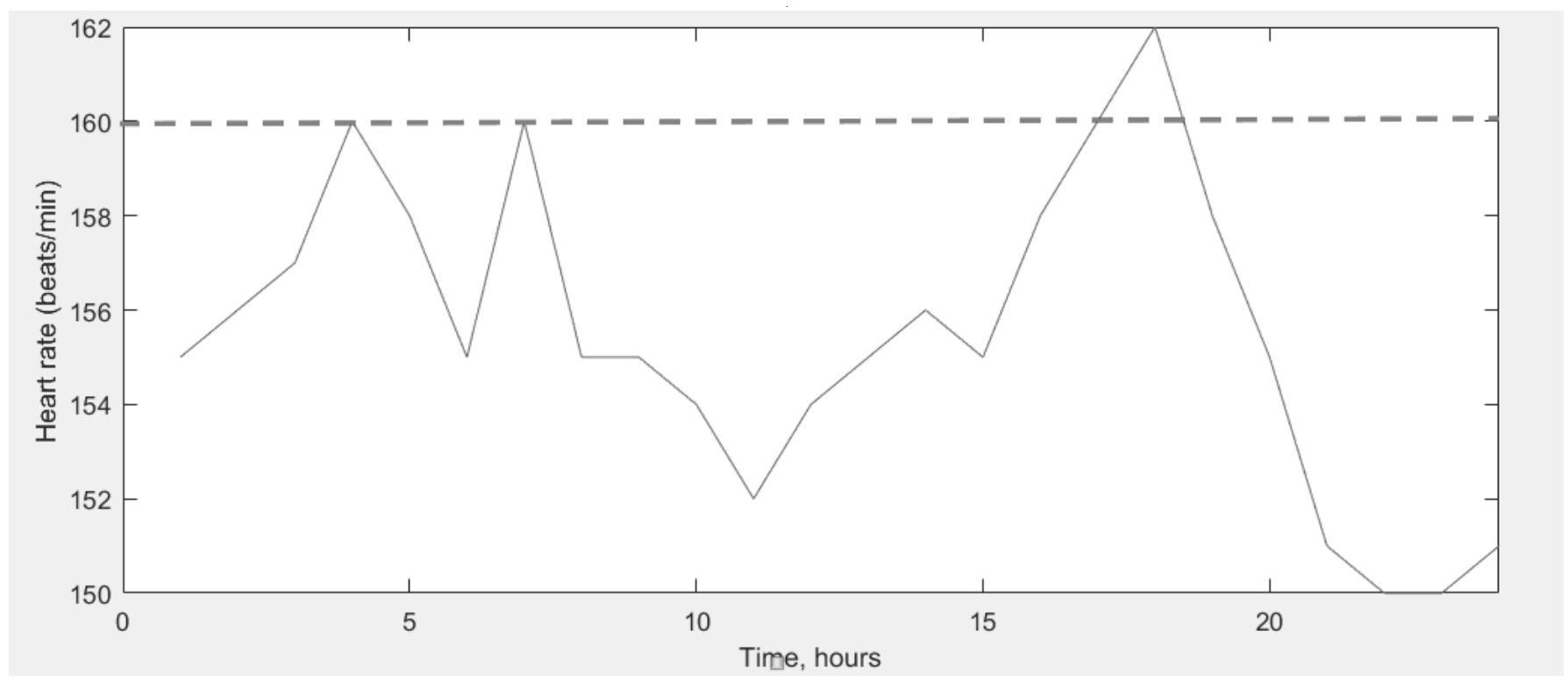

Рис. 5(c). Результаты мониторинга частоты сердечных сокращений для случая 1. Выше красной отметки посылается сигнал медицинскому работнику

телеметрии очереди сообщений используется в системе [15], где безопасность транспортного уровня и уровень защищенных сокетов используются для обеспечения защищенной связи[16].

12. Для аутентификации выполняется двухэтапная проверка. На первом этапе пользователь должен предоставить правильный идентификатор пользователя и пароль. Если предоставленная информация верна, то используется биометрическая аутентификация, где для аутентификации пользователя используется распознавание изображения сетчатки глаза или распознавание нажатия клавиш. Эта двухэтапная проверка выполняется для повышения безопасности системы, поскольку биометрические характеристики каждого пользователя уникальны[17].

Данные с датчиков могут собираться на компьютере с помощью различного программного обеспечения. В работе представлено использование MatLab/Simulink для мониторинга состояния здоровья новорожденных. Таким образом, MatLab является оптимальной системой для мониторинга здоровья и оповещения для неонатальной интенсивной терапии.

Рассмотрим результаты отражения системы мониторинга здоровья для неонатальной интенсивной терапии с использованием MatLab для нескольких примеров.

Случай 1. Ребенок весом 1600 г, возраст ребенка 2 недели. Рекомендуемая температура окружающей среды $-33^{\circ} \mathrm{C}$. Пример снятых данных и переданных в среду MatLab представлены на рисунке 5(a, 6 и с).

Случай 2. Ребенок весом 1300 г, возраст ребенка 3 дня. Рекомендуемая температура окружающей среды $-35^{\circ} \mathrm{C}$. Пример снятых данных и переданных в среду MatLab представлены на рисунке 6(a, б и c).

Таким образом, при повышенном или пониженном значении одного из параметров посылается сигнал медицинскому работнику на мобильный телефон. При использовании такой системы медицинский работник автоматически уведомляется системой о возникновении тяжелого случая, чтобы он мог предпринять необходимые действия для решения проблемы.

\section{Зак^ючение}

В статье представлена автоматизированная система неонатального мониторинга с использованием СМОВ. Использование датчиков в этой системе снижает накладные расходы, связанные с проводным подключением существующих систем. Помимо этого, система может автоматически обнаруживать переохлаждение, гипертермию, сердечные проблемы, некорректную температуру окружающей среды и информировать медицинских работников через мобильные устройства в любом месте в любое время. Хранение данных о здоровье и доступ к ним происходят внутри облака. Немедленное информирование медицинского работника о неблагоприятном случае увеличивает вероятность излечения и обеспечивает лучшее качество жизни. 


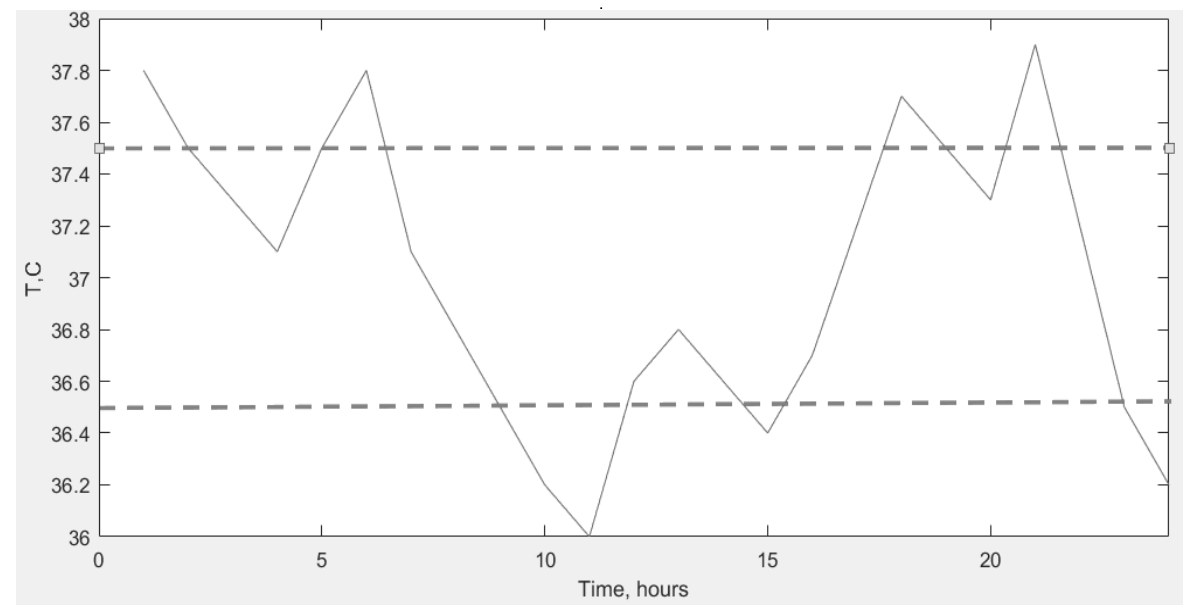

Рис. 6(а). Результаты мониторинга температуры тела ребенка для случая 2. Ниже и выше красной отметки посылается сигнал медицинскому работнику

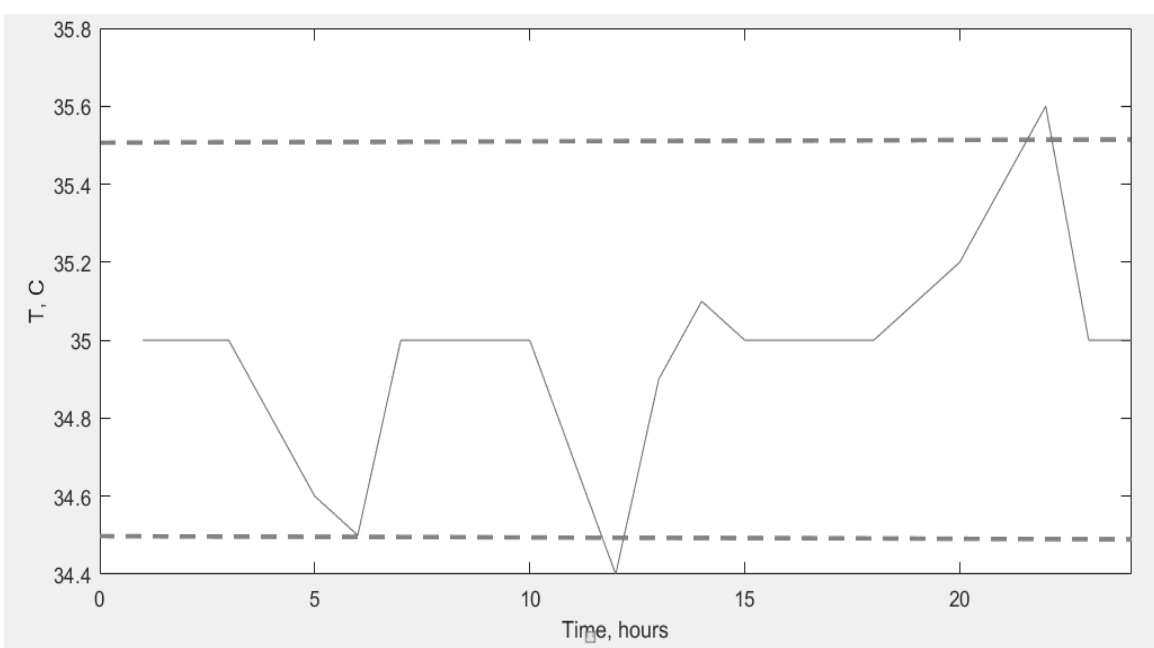

Рис. 6.(б). Результаты мониторинга температуры окружающей среды для случая 2. Ниже и выше красной отметки посылается сигнал медицинскому работнику

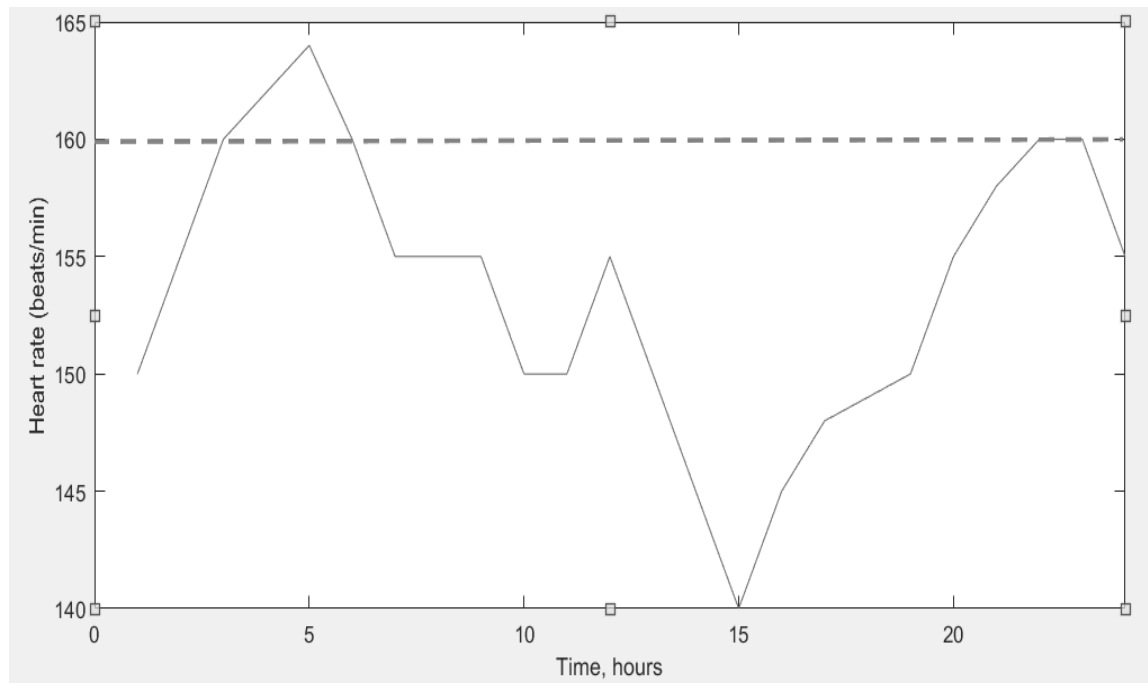

Рис. 6(с). Результаты мониторинга частоты сердечных сокращений для случая 2. Выше красной отметки посылается сигнал медицинскому работнику 


\section{ЛИТЕРАТУРА}

1. De D., Mukherjee A., Ray A., ET AL.:'A novel architecture of green sensor mobile cloud computing', IET Wirel. Sensor Syst., 2016, 6, (4), pp. 109-120.

2. Neonatal Monitoring Technologies: Design for Integrated Solutions [Электронный ресурс]: Режим доступа: https://www.igi-global.com/book/neonatalmonitoring-technologies/60777 (дата обращения: 28.09.2021).

3. Интеллектуальные навигационно-телекоммуникационные системы управления подвижными объектами с применением технологии облачных вычислений. - М.: Горячая линия - Телеком, 2014. - 158 с.

4. Zhao, W., Sun, Y. and Dai, L. Improving Computer Basis Teaching through Mobile Communication and Cloud Computing Technology. Proceedings of the 3rd International Conference on Advanced Computer Theory and Engineering (ICACTE'10), 2010.

5. Heavy Reading Real World Research. The Mobile Cloud Market Outlook to 2020, 2020.

6. К Каар, Н. Интеллектуальные навигационно-телекоммуникационные системы управления подвижными объектами с применением технологии облачных вычислений; РГГУ — Москва, 2015. - 158 с.

7. Kchaou, H., Kechaou, Z. and Alimi, A.M. Towards an Offloading Framework Based on Big Data Analytics in Mobile Cloud Computing Environments. Procedia Computer Science, 53, 2015, pp. 292-297.

8. Fernando, N., Loke, S.W. and Rahayu, W. Mobile Cloud Computing: A Survey. Future Generation Computer Systems, 29, 2013, pp. 84-106.

9. E.Z Piraniy, F. Bulakiwala, M. Kagalwala, M. Kalolwala, S. Raina, Android based assistive toolkit for alzheimer. Procedia Comput. Sci. 79:, 2016, pp. 143-151.

10. P. Verma, S.K Sood, S. Kalra, Cloud-centric loT based student healthcare monitoring framework. J. Ambient. Intell. Humanized Comput.116:, 2017, pp. 1-17

11. S. Sareen, S.K Sood, S.K Gupta, loT-based cloud framework to control ebola virus outbreak. J. Ambient. Intell. Humanized Comput.9:, 2016, pp. 1-18.

12. Z. Xia, Y. Zhu, X. Sun, Z. Qin, K. Ren, Towards privacy-preserving content-based image retrieval in cloud computing. IEEE Trans. Cloud Comput.6(1), 2018, pp. 276-286.

13. Z. Yang, Q. Zhou, L. Lei, K. Zheng, W. Xiang, An loT-cloud based wearable ECG monitoring system for smart healthcare. J. Med. Syst.40(12), 2016, pp. 1-11.

14. J.R Cheng, R.M Xu, X.Y Tang, V.S Sheng, C.T Cai, An abnormal network flow feature sequence prediction approach for DDOS attacks detection in big data environment. Comput. Mater. Continua. 55:, 2018, pp. 95-119.

15. C.R Wu, E. Zapevalova, Y.W Chen, F. Li, Time optimization of multiple knowledge transfers in the big data environment. Comput. Mater. Continua. 54:, 2018, pp. 269-285

16. H. Cheng, D. Feng, X. Shi, C. Chen, Data quality analysis and cleaning strategy for wireless sensor networks. EURASIP J. Wirel. Commun. Netw.61:, 2018, pp. $1-11$.

17. A.E Murabet, A. Abtoy, A. Touhafi, A. Tahiri, Ambient assisted living systems models and architectures: A survey of the state of the art. J. King Saud Univ. Comput. Inf. Sci.1(1), 2018, pp. 1-10. 